\title{
ARR screening using IDS-iSYS Aldosterone and Direct Renin: effects of medication in essential and renovascular hypertension, Cushing's syndrome and primary aldosteronism
}

\author{
R. Sustarsic ${ }^{1}$, C. Lonati ${ }^{2}$, J. Manolopoulou ${ }^{3}$, M. Reincke ${ }^{1}$, M. Bidlingmaier ${ }^{1}$, A. Morganti ${ }^{2}$ \\ ${ }^{1}$ Endocrine Research Unit, Medizinische Klinik und Poliklinik IV, Klinikum der Universität, Munich, Germany \\ 2 Department of Internal Medicine and Hypertension Center, San Giuseppe Hospital, University of Milan, Milan, Italy \\ 3 Immunodiagnostic Systems, Boldon, UK
}

\begin{abstract}
Background
Primary aldosteronism (PA), a more frequent form of secondary hypertension than previously thought, is commonly produced by either an aldosterone-producing adenoma or by adrenal hyperplasia. PA is characterized by elevated plasma levels of aldosterone and suppressed renin secretion. Calculation of the aldosterone-to-renin ratio (ARR) was introduced 30 years ago as a convenient screening test for the diagnosis of PA due to the unique and characteristic profile of aldosterone and renin levels observed in this disorder. The relationship between the ARR and aldosterone values can be used to easily depict subgroup differentiation of primary and secondary hyper- and hypoaldosteronism as well as normal subjects (Fig. 1; McKenna et al., 1991). By assessing the levels of plasma aldosterone in relation to $A R R$, a variety of disorders of the renin-angiotensin-aldosterone axis can be described.

Identifying hypertensive patients with PA is crucial for optimizing their treatment. Various medications used to treat hypertension are known to alter levels of aldosterone and renin, thus affecting the ARR. As withdrawing all medication before screening is often unrealistic we aimed to determine the degree of interference of some commonly used agents on the ARR using assays on the automated IDS-iSYS platform (Boldon, UK)
\end{abstract}

\section{Methods}

In order to depict the distribution of ARR values across various disease areas and to show the relationship to the levels of plasma aldosterone, we measured ARR in a total of 722 samples. These included samples from healthy individuals (262) as well as from patients with essential hypertension (282), renovascular hypertension (16), Cushing's syndrome (3) and PA (159).

To evaluate how hypertension medication affects the ARR, we focused on a cohort of 65 patients (32 males, 33 females) with essential hypertension, renovascular hypertension, PA or Cushing's syndrome (Fig. 2). Fifty-seven of the patients were taking anti-hypertensive medication at the time of screening. Mean age was $59 \pm 17$ years. For the majority of patients, both supine and standing samples were available.

IDS-iSYS Aldosterone and Direct Renin assays were used for all aldosterone and renin measurements. A previously determined ARR cut-off of greater than $1.1 \mathrm{ng} / \mathrm{dL} / \mu \mathrm{U} / \mathrm{mL}$ was used to indicate the presence of PA.

\section{Results}

The relationship between ARR and plasma aldosterone in healthy individuals as well as in patients with different types of hypertension is depicted in Fig. 3.

ARR values of samples from the cohort, in which effect of hypertension medication was evaluated, are graphed in Fig. 4. In patients with PA, 15 of 19 samples had an ARR value above the cut-off. All samples from patients with renovascular hypertension $(n=16)$ and Cushing's $(n=3)$ displayed a negative ARR regardless of medication. Importantly, there is a clear discrimination between PA and renovascular hypertension, which is the other major form of secondary hypertension. In samples from patients with essential hypertension ( $n=78$ ), 20 false positive ARRs were measured, 16 of which were standing samples. Seventeen of the 20 false positives were from patients taking $\beta$-blockers, whereas two were from patients not taking any hypertension medication.

\section{Conclusions}

The theoretical classification of patients based on the relationship between ARR and plasma aldosterone did not accurately predict the type of hypertension in our samples. The high overlap between the groups presented in Fig. 3 could be partly due to effects of hypertension medication.

Interrupting hypertension medication for ARR screening gives the most accuracy but is not ideal for the patient. We observed a false positive outcome in $26 \%$ of samples from patients with essential hypertension, which was mostly associated with $\beta$-blockers and/or standing sampling position. Therefore, it may not be necessary to withdraw all drugs for the ARR screening as long as the effects are taken into account when interpreting results.

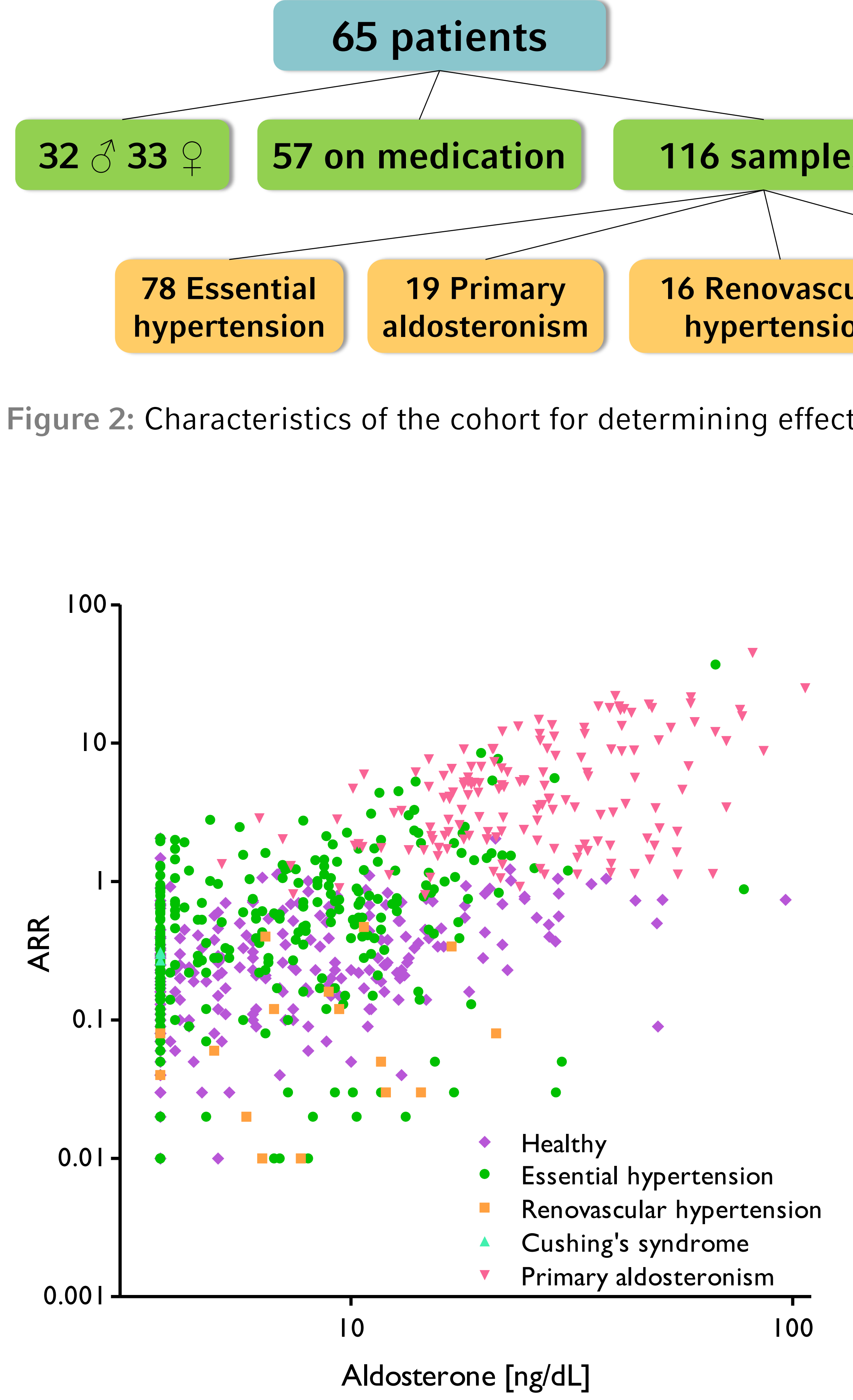

Figure 3: Relationship between ARR and plasma aldosterone values in healthy individuals and different hypertensive patient groups.

\section{$32{ }^{1} 33+57$ on medication 116 samples

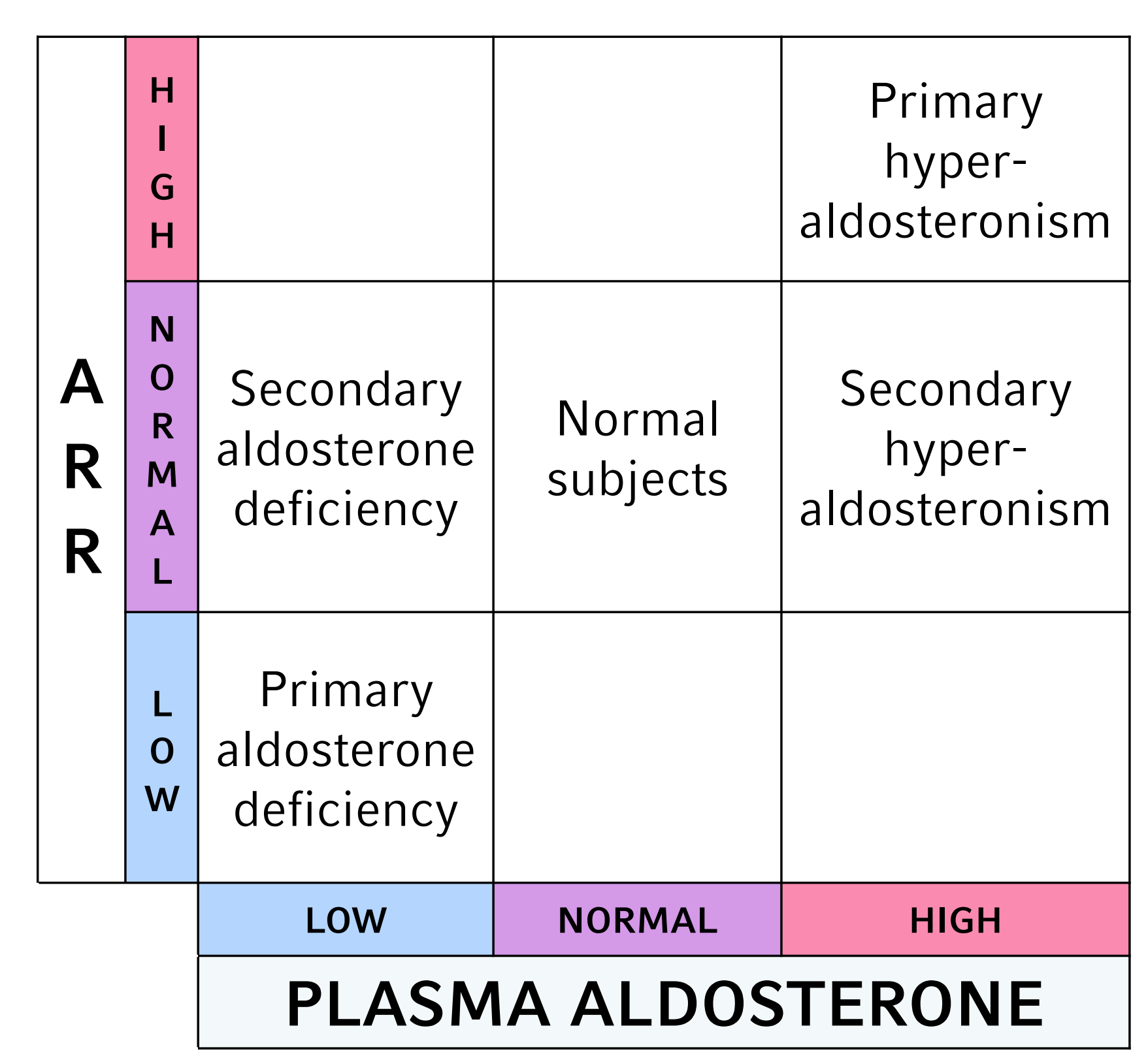

Figure 1: Theoretical classification of patients based on the relationship between ARR and plasma aldosterone values. Modified from McKenna et al., 1991.

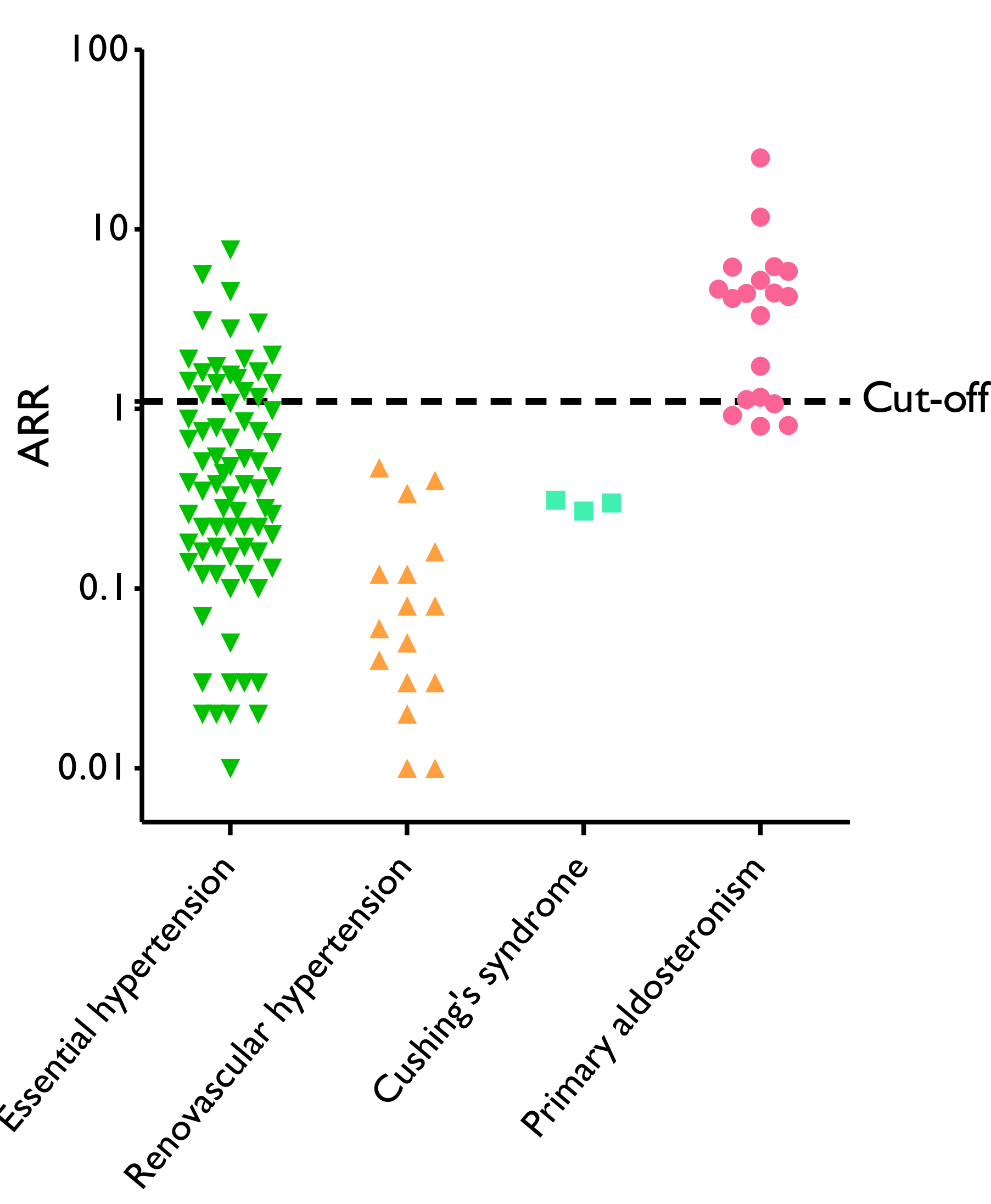

Figure 4: ARR in samples from different groups of hypertensive patients, most of whom are on hypertension medication. Dashed line marks the $1.1 \mathrm{ng} / \mathrm{dL} / \mu \mathrm{U} / \mathrm{mL}$ cut-off for PA.

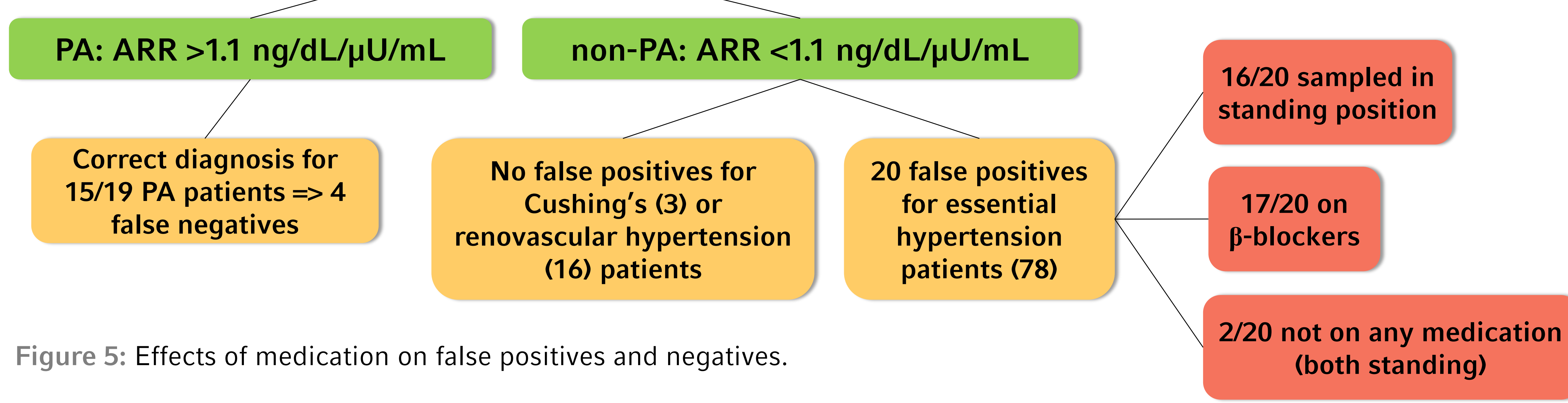

